\title{
TRANSICIÓN EN LOS REGIONALISMOS SUDAMERICANOS: DINÁMICAS DOMÉSTICAS Y FACTORES GLOBALES
}

\author{
TRANSITION In SOUth AmERICAN REgIONALISMS: DOMESTIC DyNAMICS \\ AND GLOBAL FACTORS
}

Isabel Clemente Batalla

\begin{abstract}
RESUMEN
Este artículo analiza la transición en los regionalismos sudamericanos en una fase marcada por cambios políticos en la región y en el sistema global. El análisis se fundamenta en la discusión teórica sobre regionalismos y en aportes de la investigación empírica. La primera sección considera la formación del concepto de Sudamérica como unidad espacial diferenciada. Los resultados de este estudio establecen que UNASUR atraviesa una fase de estancamiento institucional y pérdida de capacidades de ejecución de su misión como foro de cooperación intra-regional, concertación política y solución de conflictos regionales sudamericanos. En cambio, en el nivel subregional, la coordinación de políticas orientada hacia la convergencia de los dos bloques sub-regionales mayores, MERCOSUR y Alianza del Pacífico, en el breve período que examina este trabajo revela avances y planeación realista pero el logro de sus objetivos de convergencia están condicionados a la capacidad de construir consenso entre modelos de integración diferentes.
\end{abstract}

\section{PALABRAS CLAVE}

Regionalismo-UNASUR-solución de conflictos- MERCOSUR- Alianza del Pacífico convergencia

\begin{abstract}
This paper examines the transition in South American regionalisms during a period marked by political changes in several countries in the area, as well as in the global system. The analysis is framed into the theoretical discussion on regionalism, and is based upon contributions of empirical research. The first section considers the definition of the concept of South America as a distinctive spatial unit. The findings of this study show that UNASUR experiences a period of institutional stagnation and loss of capacities for fulfilling its mission as a forum for intra-regional cooperation, concerted political agreements, and South American regional conflict-solution. Conversely, at the sub-regional level, the MERCOSUR and the Pacific Alliance' efforts in policycoordination towards convergence prove advancements and realist planning during the short period covered in this paper, however, success in attaining their convergence goals is subject to their capacity for building consensus between different integration models.
\end{abstract}

\section{KEY WORDS}

Regionalism - UNASUR - conflict resolution - MERCOSUR - Pacific Alliance -convergence. 


\section{INTRODUCCIÓN}

Los regionalismos sudamericanos atraviesan una fase de cambios que se originan en parte en crisis políticas de algunos estados de la región, y en parte en la inestabilidad creada por factores de orden global. Esos cambios configuran un proceso de transición en el modelo de regionalismo que es abordado en este artículo en dos direcciones: la evolución de UNASUR, organización regional que comprende al conjunto de 12 países de América del Sur, y la trayectoria de los dos bloques subregionales mayores, MERCOSUR y Alianza del Pacífico, hacia una creciente convergencia.

La discusión teórica sobre regionalismos se ha concentrado durante la última década en las articulaciones entre sistemas regionales y sistema global. Aspectos como los sistemas de normas e instituciones o los medios y problemas de implementación no han sido incorporados en la caracterización de los modelos de regionalismos.

En ese sentido, el estudio de Mellado y Alí (2014: 186-188) aporta una perspectiva novedosa para caracterizar el regionalismo 2000-2017 por un cambio en las prioridades en detrimento de la dimensión económica y en beneficio de otras dimensiones como la política, seguridad regional, cooperación y seguridad energética, infraestructura física y participación regional. El análisis del comportamiento de esas dimensiones con base en información estadística revela que la concentración del ingreso aumentó (según cifras de CEPAL) a pesar de las altas tasas de crecimiento durante el período. El predominio de la diplomacia de cumbres y un alto protagonismo presidencial consolidó la institucionalidad mínima y el modelo intergubernamental. Por otra parte, la conducción de la política exterior de los gobiernos progresistas no produjo orientaciones ideológicas y políticas convergentes con respecto a la inserción internacional y modelos de desarrollo. Finalmente, la participación de los Estados en múltiples proyectos regionales generó superposición.

El análisis de María Izabel Mallmann (2010) también concluye que, al contrario de las expectativas creadas con el acceso de la izquierda al gobierno, en varios países de la región, el proyecto de integración sudamericano fue debilitado con la exacerbación del nacionalismo y las rivalidades y desconfianzas entre Estados. Enmarcado en la perspectiva del neo-funcionalismo, este estudio sobre el proceso de integración sudamericana examina sus resultados a partir del comportamiento de variables relativas a las condiciones específicas que favorecen la integración, agrupadas en 
cuatro dimensiones: política-ideológica, económica, social y perceptual, y relacional y contextual. En la dimensión política-ideológica, Sudamérica experimentó grados importantes de inestabilidad institucional en países como Bolivia, Venezuela y Paraguay y muy escasa coordinación de políticas públicas para aproximar a las sociedades y crear así un sustrato sólido para la integración. En la dimensión económica, los mecanismos de spill over no fueron suficientes para la reducción de las asimetrías y aumento de la interdependencia, no sólo en términos de intereses sino también de valores compartidos. Con respecto a la dimensión social y perceptual, factores como el lento y errático ritmo de la integración y la escasa previsibilidad del comportamiento de los pares generan incertidumbre y percepciones negativas sobre el futuro de la integración. Finalmente, en la dimensión relacional, algunos gobiernos no contribuyeron a la formación de percepciones positivas sobre la integración regional, mantenimiento del orden y la paz en la región. La ausencia de factores externos de cohesión regional acentuó la disgregación en el comportamiento de la región ante instancias de coyuntura (Mallmann, 2011: 57-63).

Söderbaum (2016: 40-42) propone un abordaje que integra teorías neo-funcionalistas y liberal-institucionalistas en el estudio del regionalismo y la explicación del rol de actores no estatales como los grupos de interés y los movimientos sociales, y el diseño y la acción de las instituciones regionales.

Ante un mapa confuso de superposición de modelos y patrones complejos de regionalismo, José Antonio Sanahuja (2016: 32-35) plantea un marco analítico que combina elementos de economía política internacional, perspectiva crítica en la explicación del cambio y continuidad en el regionalismo latinoamericano como respuestas a cambios en el sistema internacional, el institucionalismo histórico para analizar las variaciones específicas en cuanto al diseño institucional y las orientaciones políticas del regionalismo como contingentes a factores domésticos (objetivos de desarrollo socio-económico, instituciones, cultura política e identidad). En el estudio del ciclo regionalista entre 2005 y 2015, Sanahuja encuentra que el regionalismo latinoamericano exhibe pautas complejas que trascienden la dicotomía Atlántico-Pacífico y que, dicho ciclo, se sitúa en el contexto de la economía política internacional y sus constricciones al desarrollo socio-económico de América Latina.

La fase de transición que se inicia en 2015 y se profundiza en 2017 es resultado de una conjunción de factores de origen global y regional: en el orden global, los factores 
con mayor incidencia sobre el regionalismo sudamericano son el fin de las negociaciones para mega-acuerdos regionales, el retorno al proteccionismo en la mayor economía del mundo y el incremento de tensiones y conflictos con efectos directos en un clima de creciente inseguridad internacional; en el orden regional, las iniciativas para renegociación de tratados vigentes como el acuerdo NAFTA, las crisis políticas en países relevantes como Brasil y Venezuela y algunas medidas unilaterales por parte de Estados miembros que atentan contra acuerdos vigentes en los bloques de integración producen niveles importantes de incertidumbre y exigen nuevas respuestas en términos de estrategias regionales.

\section{SUDAMÉRICA COMO UNIDAD DE ANÁLISIS POLÍTICO}

Si bien la idea de una unión sudamericana tiene antecedentes que se remontan al siglo XIX, con la propuesta de Justo Arosemena al Congreso Hispanoamericano de Lima en 1864-65, su reaparición a inicios del tercer milenio generó dudas acerca de su viabilidad en términos económicos (Burges, 2005) y reacciones fundamentadas en la percepción de una ruptura de la unidad latinoamericana. Así, Cassio Luisielli interpreta la opción de Brasil por Sudamérica como un mecanismo de exclusión de México y como una manifestación del "enfriamiento" en las relaciones Brasil-México (Luisielli, 2010: 14-18).

Esta visión de Luisielli contrasta con las de investigadores mexicanos que identifican una línea de política exterior de México hacia el distanciamiento respecto de América Latina desde el período presidencial de Vicente Fox, seguido por una reorientación posterior hacia la recuperación de posiciones en América Latina, con el objetivo de confrontar la "amenaza" de UNASUR (Stalamachia, 2011: 100-106). Esa orientación se habría revertido posteriormente, con la gestión negociadora de México desde la presidencia del Grupo de Río, en el conflicto que enfrentó a Colombia, Venezuela y Ecuador en 2008 y con la Declaración de Salvador, Bahía, en la Cumbre de América Latina y el Caribe sobre integración y desarrollo del 16 y 17 de diciembre de 2008. La declaración de Cancún y la fundación de CELAC habrían consagrado el reintegro de México y el Caribe en el conjunto latinoamericano (Rojas Aravena, Francisco, 2011: 183-200).

Desde la perspectiva de Brasil, Paulo Fagundes Visentini (2011: 121) encuentra los orígenes de la iniciativa sudamericana de Brasil en la propuesta de ALCSA de 1993, formulada por el gobierno brasilero en respuesta a la formación de NAFTA por 
Estados Unidos y no como un proyecto para aislar a México. Posteriormente, durante el gobierno de Fernando Henrique Cardoso, se consolidó el abandono de la idea de América Latina y la adopción de la noción de Sudamérica como marco para la concertación regional de políticas de integración energética y desarrollo de infraestructura física.

En su formulación inicial, esta idea se haría efectiva con la articulación entre la Comunidad Americana de Naciones (CAN) y MERCOSUR (el cual ya había incorporado a Chile como miembro asociado).

La proyección internacional de América del Sur se afirmaría con la declaración de la región como zona de paz y con iniciativas como IBSA (India, Brasil y Sudáfrica) y BRICS (Brasil, Rusia, India, China y Sudáfrica). Estas iniciativas tienen principalmente como referencia algunas direcciones de la política exterior de Brasil pero no explican la opción por Sudamérica como una unidad espacial de análisis en los restantes países de América del Sur.

\section{REgIONALISMO SUDAMERICANO EN TRANSICIÓN}

La construcción del espacio sudamericano como ámbito para posiciones concertadas ante amenazas a la estabilidad democrática o riesgos para la integridad territorial de los Estados de la región, como también para la solución de conflictos intermésticos, se consolida en un proceso acumulativo de discusiones y acuerdos en cumbres realizadas entre agosto de 2000 hasta enero de 2008, el cual culmina con la aprobación, en esa última fecha, del tratado constitutivo de UNASUR.

Dotada de un diseño institucional de carácter intergubernamental y tal vez demasiado escueto para la diversidad y complejidad de los temas incluidos en su agenda (Gajate, 2009, pp.212-216), UNASUR aportó significativamente a la renovación del regionalismo con la incorporación de nuevos temas de contenido político en la agenda de la integración. En la primera cumbre realizada en Brasilia se abordaron cuestiones de seguridad y defensa y los problemas del consumo y tráfico de drogas. Este último tema fue planteado a partir de un enfoque integral que se apartaba del modelo de la "guerra contra las drogas" promovido desde Estados Unidos. La segunda cumbre sudamericana, reunida en 2002 en Guayaquil, aprobó la Declaración sobre Zona de Paz sudamericana por la cual se prohibió el uso de armas de destrucción masiva y se condenó el uso de la fuerza entre Estados. La tercera cumbre, celebrada en Cusco en 
2004, incluyó en la agenda el problema de la seguridad ciudadana y la necesidad de políticas públicas elaboradas con un enfoque integral, multidisciplinar y multisectorial (Sanahuja, 2014, pp. 495-496).

La gestión de crisis regionales tuvo resultados exitosos en Bolivia en 2008, con la salida del conflicto generado por movimientos separatistas en territorios del oriente boliviano y, en 2009, con la superación de la crisis entre Colombia, Venezuela y Ecuador, originada en el ataque a un campamento de las FARC establecido en la frontera norte de Ecuador, en una acción concertada con el Grupo de Río. Finalmente, la creación en 2009 del Consejo de Defensa Sudamericano como mecanismo de cooperación e intercambio de información, introdujo un área de políticas nueva en la integración latinoamericana. La declaración de zona de paz de América del Sur (ZOPACAS), establecida en 1986 por resolución de la Asamblea General de Naciones Unidas, se proponía como base para la construcción de una identidad suramericana de defensa y la formación de consensos regionales en el área de seguridad. Este esfuerzo de concertación hacia una visión estratégica común sobre temas de seguridad y defensa estuvo, sin embargo, ausente en lo que respecta a las relaciones externas del bloque sudamericano: en las relaciones exteriores de los países sudamericanos con actores del sistema global continuó dominando el tratamiento bilateral basado en el interés nacional de los Estados.

A partir de 2014, el desempeño de UNASUR revela signos importantes de desinstitucionalización e inconsistencias respecto de su mandato original: ellas tienen que ver tanto con problemas de implementación como con incapacidad para lograr los consensos políticos necesarios. A continuación, se examinan dos problemas centrales: el fracaso en el esfuerzo por cubrir la vacante en la Secretaría General y las debilidades en el tratamiento de algunos problemas regionales.

\section{LA VACANCIA EN LA SECRETARÍA GENERAL}

En 2017 emerge el problema institucional más grave de UNASUR, originado en la imposibilidad de realizar la designación del Secretario General al término del período de Ernesto Samper. Dada su estructura institucional minimalista, este hecho tiene consecuencias importantes. La Secretaría General, creada en 2010 con sede en Quito, es el organismo responsable de la ejecución de los mandatos que le confieren los órganos de UNASUR. El período de ejercicio del cargo es de dos años, prorrogable hasta seis meses adicionales por única vez. La designación del Secretario General es 
competencia del Consejo de Jefes y Jefas de Estado y de Gobierno a propuesta del Consejo de Ministros de Relaciones Exteriores. El Secretario General no puede ser sucedido por una persona de su misma nacionalidad y no puede solicitar ni recibir instrucciones de ningún gobierno o entidad ajena a UNASUR. Debe tener dedicación exclusiva, al igual que los demás funcionarios.

Las atribuciones del Secretario General incluyen: apoyar al Consejo de Jefes y Jefas de Estado y de Gobierno, al Consejo de Ministros de Relaciones Exteriores, al Consejo de Delegados, y a la Presidencia Pro Témpore, en el cumplimiento de sus funciones; proponer iniciativas y realizar el seguimiento de las directrices de los organismos de UNASUR; participar con derecho de voz y ejercer la secretaría de las reuniones de los organismos del bloque. El Secretario General debe preparar y presentar la Memoria anual de su desempeño y los informes de actuación de todos los organismos. Debe actuar como depositario de los acuerdos en el ámbito de UNASUR y disponer su correspondiente publicación. Es responsable de presentar el proyecto de presupuesto anual a la consideración del Consejo de Delegados y adoptar las medidas necesarias para su buena gestión y ejecución. Asimismo, le compete preparar proyectos de reglamentos para funcionamiento de la Secretaría General y someterlos a la discusión y aprobación de los órganos pertinentes. Debe coordinar con otras entidades de integración y cooperación de América Latina y el Caribe para el desarrollo de las actividades que le encomienden los órganos de UNASUR. Es responsable de celebrar, de acuerdo con los reglamentos, todos los actos jurídicos necesarios para la buena administración y gestión de la Secretaría General.

Esta descripción de funciones permite evaluar la importancia que reviste la designación del Secretario General y el impacto resultante del fracaso en el intento de realizar esa designación. Hasta la finalización del mandato de Ernesto Samper Pizano, la sucesión de Secretarios Generales se cumplió con regularidad, con la excepción de Néstor Kirchner, quien falleció pocos meses después de su nombramiento en 2010. Sucedieron a Kirchner la ex-Canciller de Colombia María Emma Mejía (2011-2012), el ex-Canciller venezolano Alí Rodríguez Araque (2012-2014) y Ernesto Samper (20142017). Al finalizar este último mandato se produjo la crisis por la imposibilidad de encontrar candidatos para asumir esa posición.

En febrero de 2017, Samper presentó el balance de su gestión ante los cancilleres de Ecuador, Guillaume Long, de Colombia María Ángeles Holguín, de Argentina Susana 
Malcorra, de Bolivia Fernando Huanacuni, y de Venezuela Delcy Rodríguez, pero no pudo presentar una candidatura para su sucesión. La parálisis del organismo generada por este hecho fue explicada por Samper en entrevistas de prensa como un efecto de la "fórmula venenosa del consenso" que permitiría que un país o dos ejerzan el veto contra la voluntad de las mayorías. Sin embargo, esa situación no se produjo en las anteriores elecciones, razón por la cual es preciso establecer una explicación diferente. Samper adelantó una hipótesis según la cual dos o tres países buscarían prolongar la acefalia de la Secretaría General para evitar la adopción de medidas contra Venezuela ${ }^{1}$.

Un factor de importancia en la génesis de este impasse es la proliferación de organismos regionales. Para Katalina Barreiro, Vicerrectora del Instituto de Altos Estudios Nacionales (AEN) de Ecuador, la UNASUR es una instancia que ha permitido generar espacios de debate sobre todo en temas políticos y posee mecanismos que han funcionado muy bien a nivel de los consejos de toma de decisión. En cambio, el Embajador Emilio Izquierdo, coordinador del organismo, considera preocupante la falta de decisión en la designación de un nuevo Secretario General y teme que pueda convertirse en una suerte de símbolo de la carencia de consensos y de "cierto congelamiento" en algunas de sus instancias².

Los Cancilleres de UNASUR se propusieron elegir un nuevo Secretario General en reunión extraordinaria en Quito el 31 de enero, 2017. Guillaume Long (Ecuador) definió el perfil del futuro Secretario como alguien con una posición internacional de prestigio reconocido, con capacidad de interlocutor de los mandatarios: un ex Presidente o un ex Canciller. El nuevo Secretario General debería impulsar la integración regional y asegurar "el compromiso férreo de los países miembros para que la integración sea una política de Estado, no una política de gobierno". Long informó que se habían manejado varios nombres para el cargo, según Agencia Efe ${ }^{3}$.

Los esfuerzos de Argentina para poner al frente del organismo a su embajador en Chile, José Octavio Bordó, fracasaron. La imposibilidad de acordar en la designación

1 Recuperado de www.elobservador.com.uy/unasur-el-gran-ausente-la-crisis-venezolanan1103940.

2 "A Coordinador de UNASUR le preocupa la falta de Secretario General," El Universo, Quito, 25 de julio de 2017), Recuperado de https://www.eluniverso.com/noticias/2017/07/25/nota/6298579/coordinador-unasur-le-preocupafalta-secretario-general

${ }^{3}$ Recuperado de https://www.efe.com/efe/america/política/los-cancilleres-de-la-unasur-se -danun-mes-para-proponer-al-sucesor-samper/20000035-3165524 
de un nuevo Secretario General condenó a UNASUR a la inacción. Entre tanto, el Director de Asuntos Políticos y de Defensa, Mauricio Dorfler, se hizo cargo de la coordinación del proceso que UNASUR auspicia para relanzar el diálogo gobiernooposición en Venezuela. En cambio, la Presidencia pro Témpore no ha sufrido interrupciones y en abril de 2017 Argentina asumió ese mandato por el período que culmina el 17 de abril de 2018.

\section{IV.DÉFICITS EN EL CUMPLIMIENTO DEL MANDATO}

Un segundo tipo de problemas de UNASUR tiene que ver con el desempeño del bloque en las áreas de acción de concertación política y gobernanza regional que forman parte de su mandato. En este sentido, es visible la ausencia o la débil convocatoria lograda en torno a problemas de la agenda regional. Dos casos ilustran la pérdida de dinamismo y de capacidad de propuesta de UNASUR: el proceso de paz en Colombia y la crisis política de Venezuela.

Sobre la paz en Colombia, hubo un primer pronunciamiento de UNASUR con la declaración emitida en la reunión cumbre de Lima, en noviembre de 2012, por la cual se otorgaba el "pleno apoyo" al plan de paz del gobierno colombiano ${ }^{4}$. Posteriormente, aunque el Secretario General Ernesto Samper (presidente de Colombia en el período 1994-1998) ofreció al gobierno colombiano la cooperación del organismo regional y desplegó esfuerzos por atraer el interés de los Estados miembros de UNASUR hacia una gestión de acompañamiento al proceso de paz, no obtuvo resultados sustantivos, aun cuando algunos países integrantes como Brasil y Ecuador se ofrecieron para asistir al proceso de paz con el ELN, y Venezuela y Chile participaron en los diálogos de La Habana.

En una conferencia realizada en la sede de UNASUR el 8 de abril de 2015, Samper informó que había realizado consultas a los gobiernos de Chile y Argentina sobre la posibilidad de formar una fuerza de paz regional que colaboraría en la consolidación de la paz y acompañaría el proceso de desmilitarización tras el eventual fin del conflicto colombiano, al término de los diálogos entre el gobierno y las FARC. Esta fuerza, denominada Cruz del Sur por Samper, sería una fuerza de paz sudamericana, sin ningún tipo de compromiso hegemónico, que daría un "valor agregado" al proceso de desmovilización y desarme de la guerrilla. Esta iniciativa fue presentada al

4 El Tiempo, Bogotá, 30 de noviembre, 2012. Recuperado de http://www.eltiempo.com/archivo/documento/CMS-12413441. 
presidente Santos pero el gobierno colombiano mantuvo su plan inicial que no contemplaba la gestión de UNASUR ${ }^{5}$. Posteriormente, el 29 de agosto de 2016, el Secretario General propuso una cumbre de UNASUR por la paz y la integración.

Los esfuerzos del Secretario General por sumar la participación de UNASUR a la gestión de la paz continuaron con nuevos ofrecimientos de apoyo. Su anuncio de la disposición de UNASUR para actuar como veedor fue desestimado por el gobierno colombiano que no creyó conveniente la presencia de veedores internacionales. En enero de 2016 Samper viajó a La Habana donde celebró reuniones con Raúl Castro y transmitió el agradecimiento de UNASUR a su gobierno por servir de garante del proceso de paz y ofrecer la sede para los diálogos entre gobierno colombiano y FARC. Tras la firma del acuerdo definitivo, UNASUR emitió el $1^{\circ}$ de octubre un comunicado de felicitaciones y de reafirmación de la declaración de Sudamérica como zona de paz ${ }^{6}$.

Finalmente, en su última presentación ante la reunión del Consejo de Ministros de Relaciones Exteriores de UNASUR, el $1^{\circ}$ de febrero de 2017, Samper realizó una exposición descriptiva de los últimos avances del proceso de paz y la desmovilización y entrega de armas, eventos en los cuales no pudo participar ni estar presente.

El segundo caso que revela el declive del papel de UNASUR en la superación de conflictos regionales es el de la crisis política en Venezuela. El primer intento de mediación de UNASUR entre el gobierno de Nicolás Maduro y la oposición se produjo en marzo de 2014, con el envío de una comisión ministerial a Caracas para acompañar el diálogo político, a solicitud de Maduro.

En marzo de 2015, el Secretario General realizó una visita a Caracas en un intento de acercar a las partes en conflicto. En esa ocasión, Ernesto Samper declaró que para UNASUR era esencial que se efectuaran las elecciones legislativas por ser ese el mejor medio para dirimir las diferencias. En esa visita, Samper estuvo acompañado por los Cancilleres de Brasil, Mauro Vieira, de Ecuador, Ricardo Patiño, y la Canciller de Colombia, María Ángela Holguín.

\footnotetext{
5 “¿Una misión de paz sudamericana para Colombia? En Foreign Affairs Latinoamérica, 23 de abril, 2015. Recuperado de https://www.revistafal.com 
En 2014, este mismo grupo de cancilleres había intentado mediar, apoyado en los buenos oficios del Nuncio Apostólico de Caracas, Monseñor Aldo Giordano, obteniendo resultados poco satisfactorios. Lograron solamente que el gobierno venezolano incluyera en la agenda parlamentaria la renovación parcial del Tribunal Supremo Judicial, del Consejo Nacional Electoral, la Contraloría General de la República, la Fiscalía y la Defensoría del Pueblo. Este esfuerzo fue finalmente frustrado por el arrase casi total del oficialismo en los cargos citados. En 2015, Samper informó en entrevista con Telesur que había transmitido al gobierno venezolano que UNASUR no avalaría intentos de interrumpir la democracia.

Al año siguiente, en medio del conflicto entre OEA y el gobierno venezolano, UNASUR intentó nuevamente interponer una mediación entre oposición y gobierno pero obtuvo muy escasa audiencia. En particular, la oposición venezolana dejó saber que el expresidente colombiano carecía de representatividad y credibilidad en Venezuela pues se atribuía su nombramiento en la Secretaría General a la influencia del gobierno de Maduro. En ese contexto, Samper dirigió sus esfuerzos al Papa, a quien solicitó que interpusiera sus buenos oficios. El Tiempo (Bogotá), $1^{\circ}$ de junio, 2016, "OEA y UNASUR en caminos paralelos frente a la crisis en Venezuela"7.

En junio de 2016, UNASUR impulsó una nueva estrategia: una misión mediadora entre gobierno y oposición integrada por los expresidentes José Luis Rodríguez Zapatero (España), Martín Torrijos (Panamá), y Leonel Fernández (República Dominicana) quien tenía ya sobrada experiencia en mediaciones por haber participado en una anterior gestión entre los gobiernos de Colombia y Venezuela por la crisis generada con el ataque del ejército colombiano a un campamento de las FARC en Ecuador. Esta mediación también terminó fracasando en diciembre de 2016, cuando la Mesa de la Unidad Democrática (MUD) decidió retirarse de las negociaciones alegando que el gobierno venezolano había incumplido lo pactado. En realidad, no había existido una mediación en sentido estricto ya que los mediadores no habían logrado una reunión conjunta de las dos partes y tuvieron que limitarse a escucharlas separadamente. A partir de entonces, sin Secretario general, UNASUR quedó fuera del caso. En

\footnotetext{
${ }^{7}$ Recuperado de: http://www.eltiempo.com/archivo/documento/CMS-16608799
} 
declaraciones a la prensa el 29 de julio de 2017, Samper afirmó que la intervención del organismo en Venezuela estaba "absolutamente congelada".

La iniciativa de diálogo de los ex -presidentes, que había contado con el apoyo del Papa, culminó con la entrega de sus recomendaciones al gobierno venezolano y a UNASUR. Estas incluían el documento elaborado por el expresidente Leonel Fernández sobre propuestas para el área económica.

No es posible afirmar si este fracaso de las gestiones de UNASUR es evidencia del final de la trayectoria de este organismo regional o se trata de un fenómeno coyuntural. En realidad, tampoco la OEA, con mayores recursos que la organización sudamericana, ha tenido mejor fortuna en la superación de la crisis venezolana y sus efectos en la región. Sin embargo, si se sitúa este caso en el contexto de las debilidades institucionales de UNASUR, en particular su incapacidad para cubrir el cargo de mayor jerarquía, la Secretaría General, podría ser un indicador de pérdida de influencia.

Esta situación de retroceso de UNASUR es contemporánea de la progresiva afirmación de CELAC. Este nuevo mecanismo de gobernanza y concertación regional, fundado en la reunión cumbre de Caracas en diciembre de 2011, luego de dos reuniones previas, la Cumbre de América Latina y el Caribe sobre Integración y Desarrollo (Costa de Sauípe, Bahía, diciembre de 2008), y la Cumbre de la Unidad de América Latina y el Caribe (Cancún, México, 2010), recupera la noción espacial de América Latina y en ese sentido es superadora de la segmentación de la región en varios sub-bloques (Rojas Aravena, 2010, pp. 13-22). Tendría capacidad para sustituir a UNASUR (y poner así fin al recorte espacial sudamericano) como organismo de concertación, diálogo político y cooperación, considerando su alcance regional.

Sanahuja (2016, pp. 50-51) evalúa las potencialidades de CELAC en los siguientes términos: si bien no cuenta con un tratado constitutivo y órganos permanentes (como sí es el caso de OEA y UNASUR), se basa en el acervo del Grupo de Río y posee una importante proyección externa. Ha sido elegida por la UE como su interlocutor en las relaciones con América Latina y el Caribe y desde 2012 participa en diálogos políticos con India, China, la Federación Rusa, Corea del Sur, Japón y el Consejo de

${ }^{8}$ El Comercio (Ecuador). Recuperado de: www.elcomercio.com/actualidad/unasur-ausentecrisis-venezolana-nicolasmaduro.html 
Cooperación del Golfo. Obtuvo un logro importante con la construcción del consenso regional en torno a la normalización de relaciones con Cuba y su regreso a las relaciones hemisféricas.

\section{BLOQUES SUBREgIONALES Y AVANCES EN LA CONVERGENCIA DE MODELOS}

La articulación entre grupos de integración subregionales sudamericanos es una aspiración de larga data: como ya se anotó, en los orígenes de la propuesta de una organización sudamericana, la vía elegida inicialmente fue una convergencia entre Comunidad Andina de Naciones (CAN) y MERCOSUR, para la formación de la Comunidad Sudamericana de Naciones (CSN). La primera propuesta en ese sentido fue formulada en 1993 y posteriormente, en 2002 y 2003, CAN y MERCOSUR firmaron varios acuerdos de complementación.

Sin embargo, la CAN ha sido erosionada con el surgimiento de nuevas propuestas: el Grupo de los Tres, formado por México, Colombia y Venezuela en 1989 (Serbín y Romero, 1993); la creación de ALBA (Giacalone, 2014); las posiciones enfrentadas entre Colombia y Perú, por una parte, y Bolivia y Ecuador por otra; finalmente, la creación de la Alianza del Pacífico (García, 2013; Gutiérrez, 2013, Levi Coral y Reggiardo, 2016). Adicionalmente, en 2006 Venezuela denunció el Acuerdo de Cartagena y el Acuerdo de constitución del Grupo de los Tres y en 2012 Bolivia presentó su solicitud de ingreso al MERCOSUR. En 2015, todos los Estados parte de MERCOSUR firmaron el protocolo de adhesión de Bolivia. El avance de la Alianza del Pacífico dificultaría según Arroyave (2014) las negociaciones para un acuerdo CANMERCOSUR y en cambio prepararía el escenario para una relación interregional Alianza del Pacífico-MERCOSUR. Con todo, la CAN ha mantenido activa la cooperación internacional, particularmente con la UE, y su agenda de participación ciudadana, diálogo político, seguridad, medio ambiente y ayuda al desarrollo.

La aproximación entre MERCOSUR y Alianza del Pacífico se realizó gradualmente con el ingreso de Estados miembros de MERCOSUR en calidad de miembros observadores en la Alianza del Pacífico. La primera solicitud, presentada por Uruguay en 2012, fue seguida por la de Paraguay en 2013 y la de Argentina en 2016. En noviembre de 2014, a iniciativa de la Alianza del Pacífico, el encuentro en Cartagena de Indias de los cancilleres de MERCOSUR y la Alianza del Pacífico marcó el inicio de un proceso de aproximación. Ambos bloques acordaron continuar los intercambios (Clemente, López, Telias, 2015). 
Ese mismo año, CEPAL publicaba su documento La Alianza del Pacífico y el MERCOSUR: hacia la convergencia en la diversidad que proporcionó la base para el diálogo sobre las perspectivas de cooperación entre los dos modelos de integración. El giro hacia el proteccionismo en Estados Unidos tras el cambio de gobierno en 2017 opera como un factor adicional de convergencia. El programa de reuniones MERCOSUR y Alianza del Pacífico se intensificó: en marzo de 2017 tuvo lugar en Buenos Aires una reunión técnica sobre cooperación aduanera para el intercambio de experiencias e información sobre diversos temas (gestión de riesgos, metodología de medición de tiempos de despacho, certificados de origen, seguridad aduanera, entre otros). Un mes después, los Ministros de Comercio Exterior y los Cancilleres de los dos bloques sub-regionales firmaron una hoja de ruta sobre complementación y acercamiento entre ambos, compromiso con el libre comercio y el multilateralismo. Se acordó que el Grupo de Alto Nivel de la Alianza del Pacífico y el Grupo Mercado Común de MERCOSUR continuarían las reuniones para avanzar en los temas identificados en ambos mecanismos de integración ${ }^{9}$.

El Canciller chileno Heraldo Muñoz presentó los criterios que orientan el trabajo conjunto: no se propone una fusión de dos bloques que tienen historias distintas, sino acordar sobre complementación y acercamiento "en un momento en que reina la incertidumbre a nivel internacional” ${ }^{10}$.

Finalmente, la cumbre de MERCOSUR realizada en Mendoza entre el 17 y 21 de julio marca el cierre de esta etapa. El programa incluyó un seminario con el tema "El nuevo contexto general y la convergencia entre MERCOSUR y Alianza del Pacífico" y culminó con los comunicados de los Presidentes de los Estados Parte del MERCOSUR sobre evaluación del proceso de integración regional y convergencia MERCOSUR-Alianza del Pacífico.

\section{CONCLUSIONES}

El análisis presentado en este texto revela que el regionalismo sudamericano enfrenta los desafíos de una transición compleja en la cual inciden factores de orden global y

\footnotetext{
${ }^{9}$ Recuperado de www.mrree.gub.uy/frontend/afiledownload?1,1,778,0,S,0

10 Recuperado de www.elpais.com.uy/informacion/MERCOSUR-alianza-pacifico-seacercan.html
} 
de orden regional, con un signo común de inestabilidad. El trabajo se propuso examinar dos niveles de la integración sudamericana: la organización regional conformada por los 12 países de América del Sur y el nivel subregional integrado por dos bloques con historias diferentes, MERCOSUR y Alianza del Pacífico.

El estudio de la trayectoria de UNASUR en el último mandato revela un serio deterioro institucional, manifiesto en la imposibilidad de cubrir el cargo de Secretario General y un retroceso en las capacidades de gestión de crisis políticas con efectos en la estabilidad regional. La pérdida de poder negociador por parte de UNASUR, el carácter errático de algunas propuestas de salida de conflictos y ofertas de negociación mal organizadas pueden afectar el futuro de este foro regional o conducir a su superación por CELAC, cuya fortaleza radica en su composición latinoamericana y en el reconocimiento de actores globales como la UE y países asiáticos.

Aunque el proceso subregional con la gradual aproximación entre MERCOSUR y Alianza del Pacífico tiene un desarrollo reciente, es posible concluir que la convergencia entre los dos sistemas de integración puede encontrar las bases comunes para un acuerdo que logre congregar a la mayoría de países sudamericanos, siempre que se logren los consensos necesarios y los equilibrios entre las especificidades de cada uno de los dos modelos.

\section{BiBLIOGRAFÍA}

Arroyave Quintero, M. A. (2014). La Alianza del Pacífico y el ocaso de la Comunidad Andina: hacia una nueva reconfiguración interregional en Latinoamérica: En E. Pastrana Buelvas y H. Gehring (Eds.), La Alianza del Pacífico: mitos y realidades (pp. 117-144). Cali, Colombia: Editorial Universidad Santiago de Cali.

Burges, S. (2005). Bounded by the reality of trade. Practical Limits to a South American Region. Cambridge Review of International Affairs, 18 (3), 437-454.

CEPAL (2014). La Alianza del Pacífico y el MERCOSUR: hacia la convergencia en la diversidad. Santiago de Chile, Chile. Recuperado de http://repositorio.cepal.org/bitstream/handle/11362/37304/S1420838 es.pdf?sequence $=1$ 
Clemente I., Lopez Burián, C. y Telias, D. (2015). Uruguay y la Alianza del Pacífico: ¿repensando el modelo de inserción internacional? Cuadernos sobre Relaciones Internacionales, Regionalismo y Desarrollo, 10 (19), 23-46.

Gajate R. M. (2009). Construcción institucional de la UNASUR. En N. Mellado (Ed.), MERCOSUR y UNASUR ¿Hacia dónde van? (pp. 201-248). Córdoba, Argentina: Lerner Editora.

García, J. (2013). Alianza del Pacífico ¿Hacia dónde vamos? Agenda Internacional, XX (31), 43-54. Recuperado de http://www.revistas.pucp.edu.pe/index.php/agendainternacional/article/viewfile/7727/79 $\underline{75}$

Giacalone, R. (2014). Cambios en el regionalismo de Venezuela y Colombia (19902013). Del Grupo de los Tres al ALBA y la Alianza del Pacífico. En N. Mellado (Ed.), Regionalismo latinoamericano: dimensiones actuales (pp.29-60). Córdoba, Argentina: Lerner Editora.

Gutiérrez, A. (2013). Estrategias de desarrollo divergentes: efectos sobre la integración económica entre Venezuela y Colombia. Desafíos, 25 (1), 85-127.

Leví Coral M. y Reggiardo G. (2016). La Alianza del Pacífico en el regionalismo sudamericano actual. Revista Mexicana de Política Exterior 106, 187-204.

Luiselli Fernández C. (2010). Brasil y México: el acercamiento necesario. Revista Mexicana de Política Exterior, 90, 9-50.

Mallmann M. I. (2011). Condições para a integração: qual é contribuição do Brasil na América do Sul? Século XXI. Revista de Relações Internacionais, 2 (2), 55-63.

Mallmann M. I., (2010). Análise institucionalista da integração sul-americana. Civitas, $10(1), 11-22$.

Mellado N. (2009). La integración sudamericana entre interrogantes y teorías. En N. Mellado (Ed.), MERCOSUR y UNASUR ¿Hacia dónde van? (pp.13-41). Córdoba, Argentina: Lerner Editora.

Rojas Aravena, F. (2011). Introducción. América Latina y el Caribe: la construcción de nuevos mecanismos multilaterales. En F. Rojas Aravena (Ed.), América Latina y el 
Caribe: Multilateralismo vs. Soberanía. La Comunidad de Estados Latinoamericanos y Caribeños (pp. 13-22). Buenos Aires, Argentina: Teseo. FLACSO.

Sanahuja J. A. (2016). Regionalismo e integración en América Latina: de la fractura Atlántico-Pacífico a los retos de una globalización en crisis. Pensamiento Propio, 21 (44), 29-76.

Sanahuja J. A. y Verdes-Montenegro Escánez, F. (2014). Seguridad y Defensa en Sudamérica: regionalismo, cooperación y autonomía en el marco de UNASUR. En A. Serbín, L. Martínez y H. Ramanzini (Coords), Anuario de la Integración de América Latina y el Caribe 2013-2014, Buenos Aires, Argentina: CRIES.

Serbín A. (2015). ¿Un nuevo ciclo de regionalismo latinoamericano en el siglo XXI? Desafíos y limitaciones después de la normalización de las relaciones entre Cuba y los Estados Unidos. Recuperado de iberorus.spbu.ru/uploads/serbin.pdf

Serbín, A. y Romero, C. (Eds.) (1993). El Grupo de los Tres. Asimetrías y Convergencias. Caracas, Venezuela: Instituto Venezolano de Estudios Sociales y Políticos (INVESP), Fundación Friedrich Ebert de Colombia (FESCOL), Editorial Nueva Sociedad.

Stalamacchia Ziccardi, N. (2011). México y la CELAC: recuperando la idea de América Latina. En F. Rojas Aravena (Ed.), América Latina y el Caribe. Multilateralismo vs Soberanía (pp. 99-110). Buenos Aires, Argentina: Teseo-FLACSO.

Visentini, P. F. (2011). Brasil, América del Sur y América Latina y el Caribe. Oportunidades y desafíos de la integración. En F. Rojas Aravena (Ed.), América Latina y el Caribe. Multilateralismo vs Soberanía (pp. 111-133), Buenos Aires, Argentina: Teseo-FLACSO.

Clemente Batalla Isabel: Doctora de la Universidad de Londres, Magister en Ciencia Política de la Universidad de los Andes, Bogotá. Profesora Agregada en el Programa de Estudios Internacionales de la Facultad de Ciencias Sociales, Universidad de la República. Docente de Negociación Internacional en el postgrado en Estudios Internacionales y de los cursos Sistema Internacional y Gobernanza Global en la Licenciatura en Desarrollo de la Facultad de Ciencias Sociales. Co-coordinadora con la Profesora María Izabel Mallmann de la Pontificia Universidad Católica de Río Grande do Sul del proyecto de investigación "Impacto da Agenda Binacional de Cooperação e Desenvolvimiento Fronteiriço sobre as relações entre Brasil e Uruguai", Programa CAPES-UDELAR, proceso selectivo 2011. Entre sus publicaciones recientes se 
encuentran: "Cambio y continuidad en la relación binacional Brasil-Uruguay," en: Malmann, María Izabel, y Scheider, Theresa Cristina (Organizadoras) Fronteiras e Relações Brasil Uruguai, Porto Alegre: EDIPUCRS, 2015, pp. 71-84. "Mercocidades e Integração regional" (en coautoría con María Izabel Mallmann), en Gomes, Joseli Fiorin y Corradi, Rodrigo de Souza (Orgs.) Paradiplomacia em movimiento, Porto Alegre: UniRitter, 2015, pp. 123-136. "De la 'razón de Estado' a la utopía: aproximaciones a El Príncipe de Maquiavelo", en: Instituto de Historia de las Ideas, Maquiavelo Intemporal en los 500 años de El Príncipe, Montevideo: FCUFacultad de Derecho, 2015, pp. 197-208. E-mail: Isabel.clemente@cienciassociales.edu.uy 